\title{
THE EFFECT OF ABSORPTION SYSTEMS ON COSMIC REIONIZATION
}

\author{
Marcelo A. Alvarez ${ }^{1}$ AND Tom ABel ${ }^{2}$ \\ Draft version January 20, 2012
}

\begin{abstract}
We use large-scale simulations to investigate the morphology of reionization during the final, overlap phase. Our method uses an efficient three-dimensional smoothing technique which takes into account the finite mean free path due to absorption systems, $\lambda_{\mathrm{abs}}$, by only smoothing over scales $R_{s}<\lambda_{\mathrm{abs}}$. The large dynamic range of our calculations is necessary to resolve the neutral patches left at the end of reionization within a representative volume; we find that simulation volumes exceeding several hundred $\mathrm{Mpc}$ on a side are necessary in order to properly model reionization when the neutral fraction is $\simeq 0.01-0.3$. Our results indicate a strong dependence of percolation morphology on a large and uncertain region of model parameter space. The single most important parameter is the mean free path to absorption systems, which serve as opaque barriers to ionizing radiation. If these absorption systems were as abundant as some realistic estimates indicate, the spatial structure of the overlap phase is considerably more complex than previously predicted. In view of the lack of constraints on the mean free path at the highest redshifts, current theories that do not include absorption by Lyman-limit systems, and in particular three-dimensional simulations, may underestimate the abundance of neutral clouds at the end of reionization. This affects predictions for the $21 \mathrm{~cm}$ signal associated with reionization, interpretation of absorption features in quasar spectra at $z \sim 5-6$, the connection between reionization and the local universe, and constraints on the patchiness and duration of reionization from temperature fluctuations measured in the cosmic microwave background arising from the kinetic Sunyaev-Zel'dovich effect.
\end{abstract}

Subject headings: cosmology: theory — dark ages, reionization, first stars — intergalactic medium

\section{INTRODUCTION}

On scales relevant to the large-scale structure of reionization - hundreds of Mpc - numerical calculations cannot selfconsistently track all the sources and sinks of ionizing radiation. Resolving the IGM on the small scales relevant to QSO absorption systems optically-thick at the Lyman edge, also known as Lyman limit systems (e.g., Weyman et al. 1981; Tytler 1982; Mo \& Miralda-Escudé 1996), is particularly difficult. These absorption systems were likely to have impeded the progress of reionization (e.g., Miralda-Escudé et al. 2000; Gnedin \& Fan 2006).

What are likely values for the comoving absorption system mean free path at the end of reionization, $\lambda_{\mathrm{abs}}$ ? Prochaska et al. (2009) determined the abundance of Lyman-limit absorption systems, $d N / d z=1.9[(1+z) / 4.7]^{5.2}$, over the redshift range $3.6<z<4.4$, which translates into a comoving mean free path of $\lambda_{\mathrm{abs}}=c / H(z) /(d N / d z) \sim 415 \mathrm{Mpc}$ at $z=3.7$. This dependence on redshift is steeper than reported previously (e.g., Storrie-Lombardi et al. 1994), and implies $\lambda_{\text {abs }} \propto(1+z)^{-6.7}$. However, Songaila \& Cowie (2010) extended the constraints out to $z \sim 6$, finding a much shallower dependence, $\lambda_{\text {abs }} \propto(1+z)^{-3.44}$, with a measurement of $\lambda_{\text {abs }} \simeq 34 \mathrm{Mpc}$ at $z \sim 5.7$. Comparison of the absorption spectra and hydrodynamic simulations can also constrain $\lambda_{\mathrm{abs}}$, with Bolton \& Haehnelt (2007b) reporting $20<\lambda_{\text {abs }}<50$ Mpc at $z \sim 6$. The evolution of the mean free path at $z<6$ leaves open a wide range of possible values during the reionization epoch, from several to hundreds of Mpc. Our goal is to determine the sensitivity of the history and morphology of reionization to this wide range of possible mean free paths.

\footnotetext{
Electronic address: malvarez@cita.utoronto.ca

${ }^{1}$ Canadian Institute for Theoretical Astrophysics, University of Toronto, 60 St.George St., Toronto, ON M5S 3H8, Canada

${ }^{2}$ Kavli Institute for Particle Astrophysics and Cosmology, Stanford University, Menlo Park, CA 94025, USA
}

Direct simulation of all the processes involved in reionization is not currently possible. Theoretical studies focus either on the small scale hydrodynamics and radiative transfer (e.g., Miralda-Escudé et al. 2000; Gnedin 2000; Haiman et al. 2001; Shapiro et al. 2004; Ciardi et al. 2006; Pawlik et al. 2009; Raicevic \& Theuns 2011; McQuinn et al. 2011), or on the large scale morphology of reionization (e.g., Abel \& Wandelt 2002; Furlanetto et al. 2004; Iliev et al. 2006; McQuinn et al. 2007; Trac \& Cen 2007; Shin et al. 2008; Friedrich et al. 2011). On the largest scales, it has been somewhat surprising how well simplified "semi-numerical" approaches (e.g., Zahn et al. 2007; Mesinger \& Furlanetto 2007; Thomas et al. 2009; Choudhury et al. 2009; Alvarez et al. 2009; Santos et al. 2010; Zahn et al. 2011a; Mesinger et al. 2011a) match much more computationally expensive radiative transfer techniques (e.g., Abel et al. 1999; Gnedin \& Abel 2001; Sokasian et al. 2001; Abel \& Wandelt 2002; Iliev et al. 2006; McQuinn et al. 2007; Trac \& Cen 2007). In order to efficiently survey the wide parameter space of $\lambda_{\text {abs }}$ and focus on the large scale morphology and overall progress of reionization, we have chosen the simplified semi-numerical approach. Our implementation is similar to that of Zahn et al. (2007), with the the addition of a treatment of photon consumption in absorption systems. Our approach, in which we treat abundance of absorbers as an input parameter, is complementary to that taken by Crociani et al. (2011), where the semi-numerical approach was used to determine the large scale distribution of absorbers, rather than to model the effect of the absorbers on the progress of reionization, as we do here.

Our results are parametrized by different values of for the minimum source halo mass, ionizing efficiency of collapsed matter, and the absorption system mean free path. We describe our simulations in $\S 2$, followed by the main results in $\S 3$, ending with a discussion in $\S 4$. All calculations were done using $\left(\Omega_{m}, \Omega_{\Lambda}, h, \sigma_{8}, n_{s}\right)=(0.27,0.73,0.72,0.8,0.96)$, consistent with WMAP 7-year data (Komatsu et al. 2011). All dis- 
TABLE 1

SIMULATION PARAMETERS

\begin{tabular}{|c|c|c|c|c|c|c|c|c|}
\hline$\tau_{\mathrm{es}}$ & $\begin{array}{l}M_{\min } \\
{\left[M_{\odot}\right]}\end{array}$ & $\begin{array}{c}\lambda_{\mathrm{abs}} \\
{[\mathrm{Mpc} / \mathrm{h}]}\end{array}$ & $\begin{array}{c}t_{\mathrm{ev}} \\
{[\mathrm{Myr}]}\end{array}$ & $\zeta$ & $z_{1 / 2}$ & $z_{0.9}$ & $z_{\mathrm{ov}}$ & $\begin{array}{c}\lambda_{0} \\
{[\mathrm{Mpc}]}\end{array}$ \\
\hline 0.06 & $10^{8}$ & 8 & - & 20 & 8.1 & 5.9 & 4.3 & 87 \\
\hline 0.06 & $10^{8}$ & 32 & - & 16 & 8 & 6.3 & 5 & 181 \\
\hline 0.06 & $10^{8}$ & 256 & - & 14.5 & 8 & 6.8 & 6.2 & 294 \\
\hline 0.06 & $10^{9}$ & 8 & - & 78 & 8.2 & 6.6 & 5.6 & 98 \\
\hline 0.06 & $10^{9}$ & 32 & - & 60 & 8.1 & 6.8 & 6 & 210 \\
\hline 0.06 & $10^{9}$ & 256 & - & 40 & 8.1 & 7.24 & 6.9 & 375 \\
\hline 0.09 & $10^{8}$ & 8 & - & 137 & 11 & 9.2 & 8 & 94 \\
\hline 0.09 & $10^{8}$ & 32 & - & 110 & 10.9 & 9.5 & 8.5 & 201 \\
\hline 0.09 & $10^{8}$ & 256 & - & 98 & 10.9 & 9.9 & 9.5 & 345 \\
\hline 0.09 & $10^{9}$ & 8 & - & 1200 & 11.1 & 9.8 & 9.1 & 105 \\
\hline 0.09 & $10^{9}$ & 32 & - & 920 & 11 & 10 & 9.3 & 232 \\
\hline 0.09 & $10^{9}$ & 256 & - & 820 & 11 & 10.3 & 10 & 443 \\
\hline 0.12 & $10^{8}$ & 8 & - & 1050 & 13.5 & 12.5 & 11.1 & 99 \\
\hline 0.12 & $10^{8}$ & 32 & - & 850 & 13.5 & 12.2 & 11.4 & 216 \\
\hline 0.12 & $10^{8}$ & 256 & - & 750 & 13.4 & 12.6 & 12.2 & 396 \\
\hline 0.12 & $10^{9}$ & 8 & - & $2.2 \mathrm{e} 4$ & 13.5 & 12.5 & 11.9 & 109 \\
\hline 0.12 & $10^{9}$ & 32 & - & $1.7 \mathrm{e} 4$ & 13.5 & 12.6 & 12.1 & 248 \\
\hline 0.12 & $10^{9}$ & 256 & - & $1.5 \mathrm{e} 4$ & 13.5 & 12.9 & 12.7 & 500 \\
\hline $0.09^{\mathrm{a}}$ & $10^{8}$ & 8 & - & 102 & 10.9 & 9.7 & 8.3 & - \\
\hline $0.09^{\mathrm{a}}$ & $10^{8}$ & 32 & - & 94 & 10.8 & 9.9 & 9.6 & - \\
\hline $0.09^{a}$ & $10^{8}$ & 256 & - & 92 & 10.8 & 10 & 9.8 & - \\
\hline 0.06 & $10^{8}$ & - & 10 & 15 & 8.2 & 6.9 & 6.4 & 300 \\
\hline 0.06 & $10^{8}$ & - & 50 & 15 & 8.1 & 6.4 & 5.6 & 264 \\
\hline 0.06 & $10^{8}$ & - & 100 & 15 & 8.0 & 6.1 & 5.3 & 170 \\
\hline
\end{tabular}

${ }^{a}$ Obtained using sharp $k$-space filtering

tances are comoving.

\section{SIMULATIONS}

Table 1 shows our simulation parameters: $\zeta$, the ionizing efficiency, $\lambda_{\text {abs }}$, the comoving absorption system mean free path, $t_{\mathrm{ev}}$, the photoevaporation time for the evolving $\lambda_{\text {abs }}$ models, and $M_{\min }$, the minimum halo mass of galaxies. Also shown are the redshifts when the global ionized fraction equals $0.5,0.9$, and $1-z_{0.5}, z_{0.9}$, and $z_{\mathrm{ov}}$, respectively, and the bubble mean free path when the neutral fraction is $0.1, \lambda_{0} \equiv$ $\lambda_{\mathrm{b}}\left(x_{\mathrm{HI}}=0.1\right)$. The parameters were varied to give a Thomson scattering optical depth of $\left(\tau_{-}, \tau_{0}, \tau_{+}\right)=(0.06,0.09,0.12)$, corresponding to the 2- $\sigma$ constraint from WMAP (Komatsu et al. 2011), with the He I fraction tracking $\mathrm{H} \mathrm{I}$, and instantaneous He II reionization at $z=3$. All simulations used $4096^{3}$ cells in a $2 \mathrm{Gpc} / h$ box.

\subsection{Mean reionization history}

We model the mean reionization history wtih two spatially uniform mean free paths: that corresponding to ionized bubbles, $\lambda_{\mathrm{b}}$, and Lyman-limit systems, $\lambda_{\mathrm{abs}}$. Ionizing radiation is attenuated by their superposition, so that $\lambda_{\mathrm{mfp}}^{-1}=\lambda_{\mathrm{b}}^{-1}+\lambda_{\mathrm{abs}}^{-1}$. The spatially averaged ionizing flux is $F(z)=\lambda_{\operatorname{mfp}}(z) \epsilon(z)$, where $\epsilon(z)$ is the ionizing photon emissivity. We set $\epsilon(z)=$ $\zeta n_{H, 0} \dot{f_{\text {coll }}}(z)$, where $\zeta$ is number of ionizing photons per collapsed hydrogen atom, corrected for recombinations outside of absorption systems. The mean ionization rate is

$$
\frac{d x}{d t}=\zeta \dot{f}_{\text {coll }}(z) \frac{\lambda_{\text {abs }}(z)}{\lambda_{\text {abs }}(z)+\lambda_{\mathrm{b}}(x)} .
$$

When $\lambda_{\mathrm{abs}} \gg \lambda_{\mathrm{b}}$, photons typically reach the edges of bubbles without being absorbed by intervening Lyman-limit systems, and the photoionization rate is equal to the emission rate. When $\lambda_{\text {abs }} \ll \lambda_{\mathrm{b}}$, the probability of a photon reaching

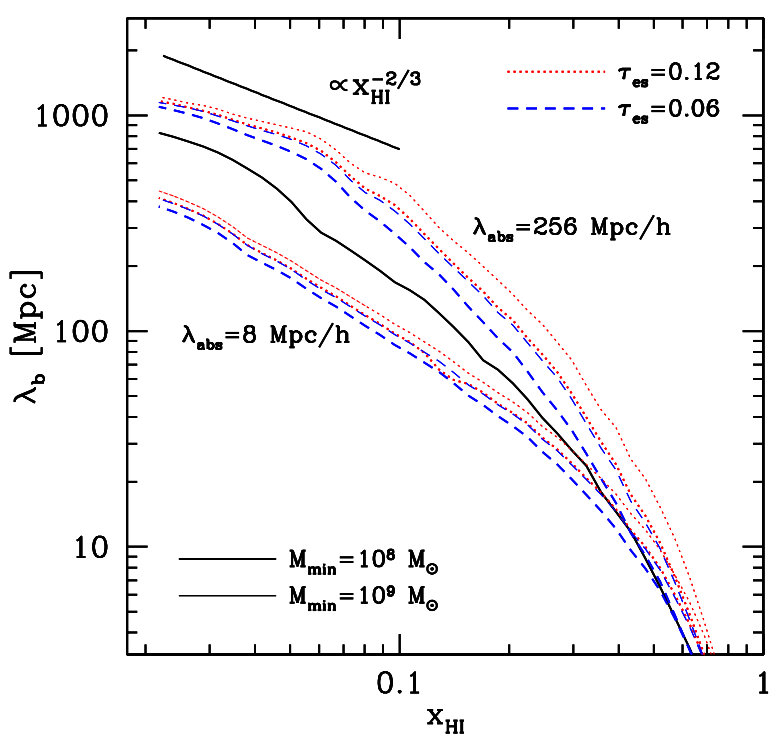

FIG. 1.- Bubble mean free path, $\lambda_{\mathrm{b}}$, as a function of mean neutral fraction, $x_{\mathrm{HI}}$. All models exhibit a characteristic scale of $\sim 10 \mathrm{Mpc}$ at the halfionized epoch. During percolation, lower values of $\lambda_{\text {abs }}$ lead to lower values of $\lambda_{\mathrm{b}}$. The solid line corresponds to the evaporating minihalo model with $t_{\mathrm{ev}}=100 \mathrm{Myr}$. At $x_{\mathrm{HI}}<0.1$ the relation approaches approximately $\lambda_{\mathrm{b}} \propto x_{\mathrm{HI}}^{-2 / 3}$.

the edge of a bubble is $\lambda_{\text {abs }} / \lambda_{\mathrm{b}}$, and the ionization rate is suppressed.

A more realistic treatment would involve allowing $F, \lambda_{b}$, and $\lambda_{\text {abs }}$ to vary spatially, obtaining a spatially-dependent solution of equation (1), which can then be averaged to find the global reionization history. We have chosen the simpler uniform model of equation (1) as a starting point. Although this choice does not affect our results on the morphology of the ionization field at fixed ionized fraction, the assumptions underlying equation (1) should be kept in mind when interpreting the reionization history and photon consumption rates we find.

\subsection{Spatial variations}

Our three-dimensional model is based on that of Furlanetto et al. (2004), later extended to three dimensions by Zahn et al. (2007). Its main assumption is that a region is fully ionized if its collapsed fraction is greater than some threshold, $\zeta f_{\text {coll }}>1$. As shown in Alvarez et al. (2009), by smoothing the linear density field over a range of scales, one can efficiently determine when each point is first reionized, $z_{r}$. We do not smooth over scales with radii larger than $\lambda_{\text {abs }}$, since absorption systems shield radiation from these distances. We assume that the absorption systems contribute a spatially uniform opacity, and therefore the scale beyond which we do not smooth is the same everywhere.

This approach is desirable because the order in which points are ionized is the same as that in which the Furlanetto et al. (2004) criterion is first met around each point. However, the reionization redshifts do not generally result in the correct average ionization fraction for a sharp smoothing filter in real space (see below for solutions using a sharp filter in $k$-space, which does conserve photons; Zahn et al. 2007). To obtain a self-consistent solution, we determine a correction to the reionization redshift at each point, $z_{r, c}\left(z_{r}\right)$, by matching the simulated reionization history to the global reionization his- 


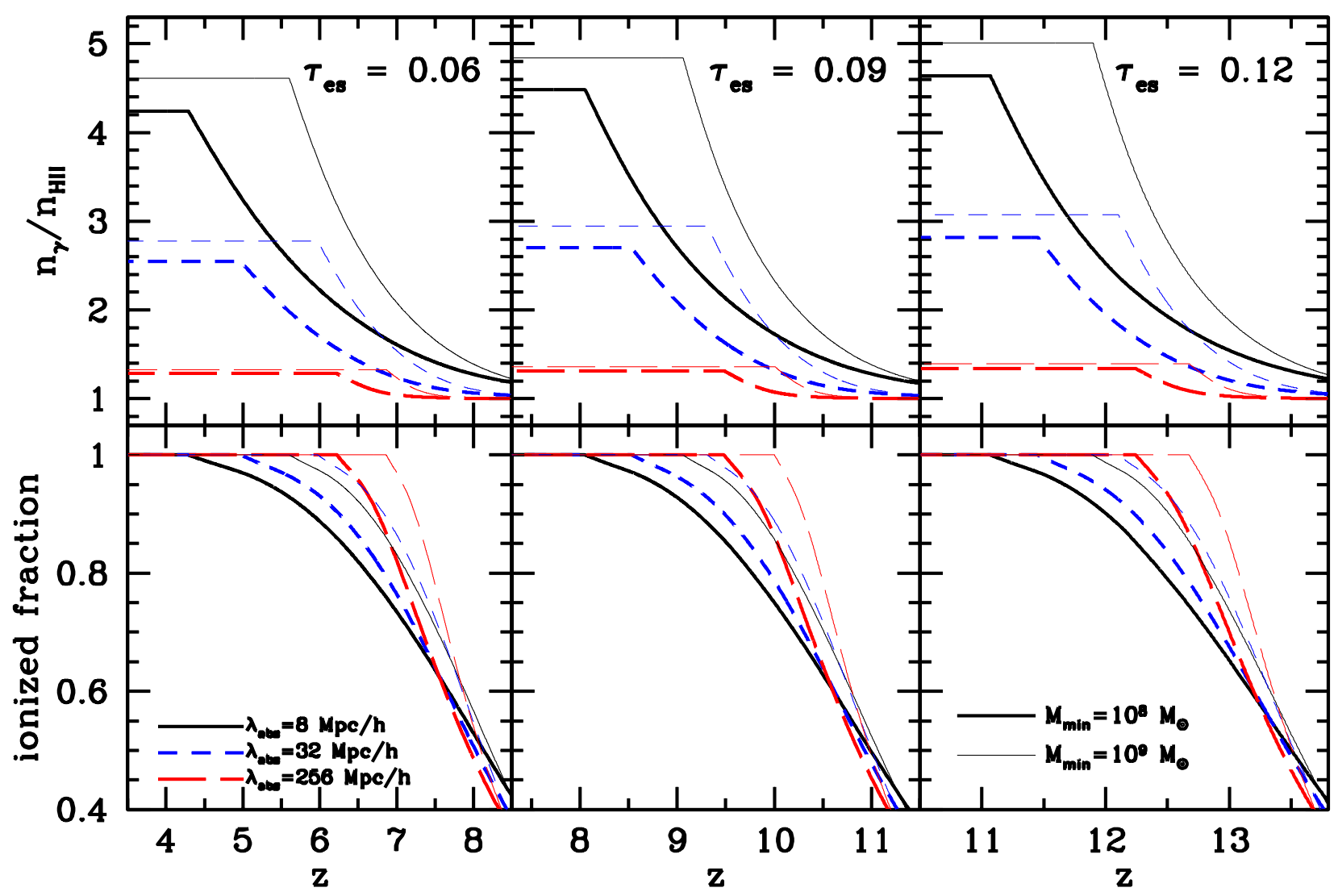

FIG. 2.- Global reionization histories (bottom) and photon consumption (top). Different colors indicate different values of $\lambda_{\text {abs }}$, while the panels indicate different Thomson scattering optical depths, $\tau_{\mathrm{es}}=0.06,0.09$, and 0.12 , from left to right. Note that in all cases the early part of the reionization history is insensitive to $\lambda_{\mathrm{abs}}$, when the bubbles are relatively smaller. Lower values of $\lambda_{\mathrm{abs}}$ delay the end of reionization.

tory,

$$
\int_{z_{r}}^{\infty} \frac{d p}{d z} d z=\int_{z_{r, c}}^{\infty} \frac{d x}{d t} \frac{d t}{d z} d z
$$

where $d p / d z$ is the distribution of uncorrected simulated reionization redshifts, and $d x / d t$ is given by equation (1). This calibration procedure does not change the overall topology we find at fixed ionized fraction, but does change the overall reionization history.

We calculate $\lambda_{\mathrm{b}}(x)$ by random raytracing. If a ray starts in an ionized region, it is extended in a random direction until it reaches a neutral cell. The bubble mean free path is then the average over the lengths of all such rays. The average converges with on the order of $10^{4}$ rays.

About 30 logarithmically-spaced smoothing scales are sufficient to converge on the reionization morphology. Because we determine the reionization redshifts simultaneously, the amount of data stored and the operation count are greatly reduced compared to determining the ionization field at each redshift. Since the assumption that points are either highlyionized or neutral is made in both cases, no information is lost by following the more efficient approach.

\subsection{Sharp $k$-space filtering}

Using a sharp $k$-space filter leads to a method which does conserve photons (Zahn et al. 2007) in the long mean free path limit, at the expense of using an unphysical filter which "rings" in real space, and an ambiguity in the assignment of a smoothing scale $R_{f}$ to the filter $k_{f}$ - particularly troubling given that we want to exclude scales $R_{f}>\lambda_{\text {abs }}$. However, for comparison we have calculated a few cases with sharp $k$-space filtering, shown in Table 1.

The ionization history is found by integrating a diffusion equation for the distribution in density of points that have not crossed the barrier (Bond et al. 1991),

$$
\frac{\partial \Pi(\Lambda, \tilde{\delta}, z)}{\partial \Lambda}=\frac{1}{2} \frac{\partial^{2} \Pi}{\partial \tilde{\delta}^{2}}-\frac{\partial \tilde{\delta}}{\partial \Lambda} \frac{\partial \Pi}{\partial \tilde{\delta}},
$$

with boundary condition $\Pi(\Lambda, 0, z)=0$, where $\tilde{\delta} \equiv B(\Lambda, z)-$ $\delta, \delta$ is the density contrast, $B(\Lambda, z)=\delta_{c}(z)-\operatorname{erf}^{-1}(1-$ $\left.\zeta^{-1}\right) \sqrt{2\left(\sigma_{\min }^{2}-\Lambda\right)}$ is the bubble barrier (Furlanetto et al. 2004), and $\sigma_{\min }^{2}$ is the variance of the $z=0$ linear density field at the scale of $M_{\min }$, with the growth of structure encoded in the time dependence of $\delta_{c}(z)$.

We smooth only over scales larger than $\lambda_{\text {abs }}$ by starting integration of equation 3 at $\Lambda_{i} \equiv \sigma^{2}\left(\lambda_{\mathrm{abs}}\right)$ - the variance on the scale of the mean free path is matched to the one obtained using a top-hat filter in real space - with initial condition

$$
\Pi\left(\Lambda_{i}, \tilde{\delta}, z\right)=\frac{1}{\sqrt{2 \pi \Lambda_{i}}} \exp \left[-\frac{\left(\tilde{\delta}-B\left(\Lambda_{i}, z\right)\right)^{2}}{2 \Lambda_{i}}\right] .
$$

The total ionized fraction is obtained by integrating over the distribution at $\Lambda=\sigma_{\min }^{2}$,

$$
x(z)=1-\int_{0}^{\infty} \Pi\left(\sigma_{\min }^{2}, \tilde{\delta}, z\right) d \tilde{\delta} .
$$


If the initial condition for $\Pi$ is specified at $\Lambda=0$, (i.e. starting at a scale $\rightarrow \infty$ ), then this corresponds to smoothing over all scales, and

$$
x(z)=\zeta \operatorname{erfc}\left[\delta_{c}(z) / \sqrt{2 \sigma_{\min }^{2}}\right]=\zeta f_{\text {coll }}(z) .
$$

\subsection{Evolving $\lambda_{\mathrm{abs}}$ due to minihalos}

To model an evolving value of $\lambda_{\text {abs }}$, we assume that the absorption systems are minihalos(e.g., Abel \& Mo 1998). Given that the halo cross section increases as $M^{2 / 3}$, while $d n / d \ln M \propto M^{-1}$, small objects should dominate the mean free path, subject to photoevaporation effects (e.g., Haiman et al. 2001; Shapiro et al. 2004).

Consider a uniform distribution of dark matter halos with a number density $n_{h}(z) \equiv d n / d \ln M_{h}$. We will assume that in neutral regions, halos at mass $M_{h}$ have gas fractions of unity and would serve as Lyman-limit absorptions systems, while in ionized regions, such halos survive for a time $t_{\mathrm{ev}}$ before being evaporated by the ionizing background. The number of absorption systems evolves according to

$$
\frac{d n_{\mathrm{abs}}}{d z}=\frac{d \ln x}{d z}\left(n_{\mathrm{h}}-n_{\mathrm{abs}}\right)+\xi_{\mathrm{ev}}(1+z)^{-5 / 2} n_{\mathrm{abs}}(z),
$$

where $\xi_{\mathrm{ev}} \equiv\left(H_{0} \Omega_{\mathrm{m}}^{1 / 2} t_{\mathrm{ev}}\right)^{-1} \simeq 260\left(t_{\mathrm{ev}} / 100 \mathrm{Myr}\right)^{-1}$. We choose $M_{h}=5.7 \times 10^{3} M_{\odot}[(1+z) / 10]^{3 / 2}$, corresponding to the cosmological Jeans mass, and consider three models, in which $t_{\mathrm{ev}}=10,50$, and $100 \mathrm{Myr}$. These timescales are relatively long compared with the rather short photoevaporation times for low-mass halos found by Shapiro et al. (2004). However, if reionization is "photon-starved", as observations are suggesting (e.g., Bolton \& Haehnelt 2007b), then the flux could be low at the end of reionization, leading to longer evaporation times.

The mean free path is

$$
\lambda_{\mathrm{abs}}^{-1}(z)=\pi R_{\mathrm{vir}}^{2}(z) n_{\mathrm{abs}}(z),
$$

where $R_{\text {vir }}$ corresponds to the halo virial radius. However, the boundaries of the absorption systems are not well-defined due to hydrodynamic effects and departures from spherical symmetry. For our purposes, the minihalo model we describe here is sufficient to incorporate the effect of an evolving absorption system mean free path in our calculations. More accurate modeling of absorption systems during reionization will need to incorporate the radiative transfer of ionizing radiation in cosmological hydrodynamics simulations which resolve the Jeans mass.

We start with an initial guess for $\lambda_{\text {abs }}(z)$, only allowing points to cross the barrier at some scale and redshift if that scale is below the current value of $\lambda_{\text {abs. }}$. The values obtained for $\lambda_{b}(x)$ from the simulated ionization field are then fed back into equations 1,7 , and 8 , and the process is reapeated until convergence is reached.

\section{RESULTS}

Fig. 1 shows the bubble mean free path as a function of the neutral fraction for several parameter choices. At the half-ionized epoch, all models exhibit a characteristic bubble scale of $\lambda_{\mathrm{b}} \simeq 10 \mathrm{Mpc}$, with models with rarer sources (i.e. increasing efficiency of ionizing radiation) leading to somewhat larger bubbles. During percolation, most of the variation in $\lambda_{\mathrm{b}}\left(x_{\mathrm{HI}}\right)$ comes from variation of $\lambda_{\mathrm{abs}}$, which is most pronounced at $x_{\mathrm{HI}} \leq 0.2$. The large values of $\lambda_{b}$ indicate that

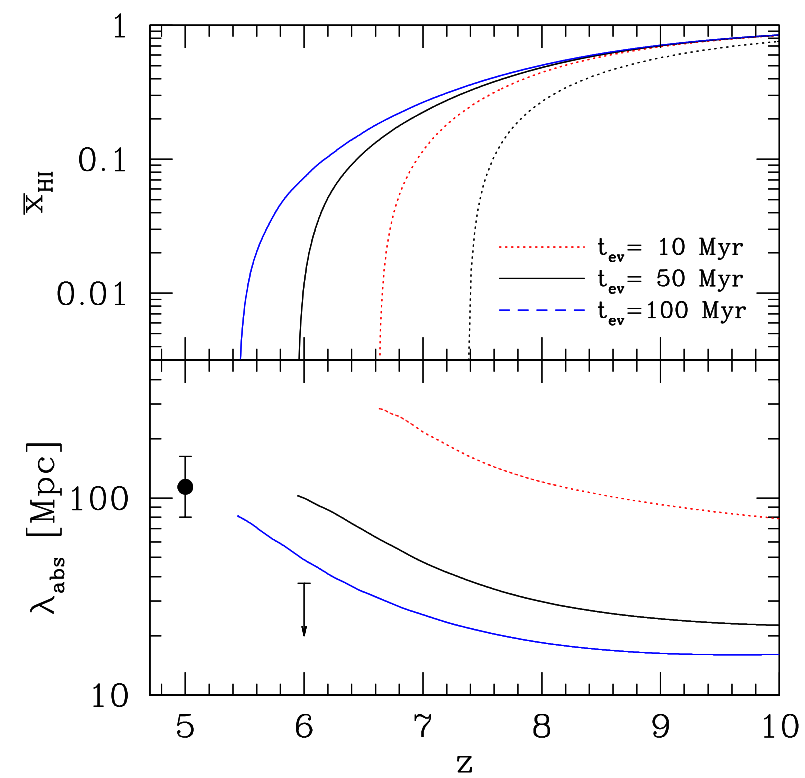

FIG. 3.- (top) Neutral fraction vs. redshift for the three evolving $\lambda_{\text {abs }}$ models considered here, in addition to the reference case, for which $\lambda_{\text {abs }} \rightarrow \infty$ (right-most dotted curve). (bottom) Absorption mean free path vs. redshift. As the last neutral patches are cleared away, so are the last reservoirs of cold neutral gas in which the minihalos can survive as absorption systems, so $\lambda_{\text {abs }}$ increases at the end of reionization. As the evaporation time decreases, the destruction rate of absorption systems increases, leading to longer mean free paths. Also shown are the constraints on $\lambda_{\mathrm{abs}}$ from Bolton \& Haehnelt (2007b) at $z=5$ and 6 .

simulation volumes in excess of several hundred Mpc on a side are necessary in order to properly model the last ten per cent of the reionization process. At $x_{\mathrm{HI}}<0.1, \lambda_{b} \sim x_{\mathrm{HI}}^{-2 / 3}$, in agreement with the model of Miralda-Escudé et al. (2000), in which a constant number of neutral clouds decrease in size at the same fractional rate at the end of reionization.

The reionization history for all of the fixed $\lambda_{\text {abs }}$ models is shown in Fig. 2. The early evolution is not sensitive to $\lambda_{\mathrm{abs}}$, because the photon mean free path is determined by the size of the ionized bubbles themselves. At later times, when the bubble sizes become comparable to $\lambda_{\mathrm{abs}}$, the reionization history is sensitive to $\lambda_{\mathrm{abs}}$, as can be seen by comparing the evolution for the bracketing values of $8 \mathrm{Mpc} / \mathrm{h}$ and $256 \mathrm{Mpc} / \mathrm{h}$. For the $\lambda_{\text {abs }}=8 \mathrm{Mpc} / \mathrm{h}$ case, the redshift at which the overlap occurs is delayed by $\Delta z \sim 1.5$, relative to $\lambda_{\text {abs }}=256 \mathrm{Mpc} / \mathrm{h}$, for $\tau_{\mathrm{es}}=0.09$ (see also the " $z_{\mathrm{ov}}$ " entries in Table 1 ).

Also shown in Fig. 2 is the number of ionizing photons per ionized atom,

$$
\frac{n_{\gamma}(z)}{n_{\mathrm{HII}}(z)}=\frac{\zeta f_{\mathrm{coll}}(z)}{x(z)}=1+\frac{1}{x(z)} \int_{0}^{x(z)} \frac{\lambda_{\mathrm{b}}\left(x^{\prime}\right)}{\lambda_{\mathrm{abs}}\left(x^{\prime}\right)} d x^{\prime} .
$$

As $\lambda_{\text {abs }} \rightarrow \infty, x \rightarrow \zeta f_{\text {coll }}$ and $n_{\gamma} / n_{\mathrm{HII}} \rightarrow 1$. We only count photons up to the moment of overlap $(x=1)$, as we are concerned here with the consumption of ionizing photons during the reionization process. This integral converges, since at the end of reionization we expect $\lambda_{b}(x) \propto(1-x)^{-2 / 3}$ as the remaining diffuse neutral hydrogen patches dissappear. For $\lambda_{\mathrm{abs}}=8 \mathrm{Mpc} / \mathrm{h}$, about 3 photons per atom are consumed in the absorption systems by $z_{\mathrm{ov}}$. In the longest mean free path case, $\lambda_{\mathrm{abs}}=256 \mathrm{Mpc} / \mathrm{h}$, the corresponding fraction is about a half.

Shown in Fig. 3 is the evolution in neutral fraction and absorption system mean free path for the three minihalo absorption system models that we simulated. Models with 
longer evaporation times lead to a higher abundance of absorption systems, and hence shorter mean free paths and a relative delay in the time of overlap. The mean free path in the $t_{\mathrm{ev}}=100 \mathrm{Myr}$ model evolves from about $40 \mathrm{Mpc}$ when $x_{\mathrm{HI}} \sim 0.1$, to about $80 \mathrm{Mpc}$ at overlap. This model is also plotted as the solid black line in Fig. 1, which shows that the instantaneous $\lambda_{\mathrm{b}}$ values for the evolving $\lambda_{\mathrm{abs}}$ model roughly interpolate between those for the fixed $\lambda_{\text {abs }}$ models. This can also be seen by noting that $\lambda_{0} \equiv \lambda_{\mathrm{b}}\left(x_{\mathrm{HI}}=0.1\right)$ for the evolving mean free path model, for which $\lambda_{\mathrm{abs}}\left(x_{\mathrm{HI}}=0.1\right) \simeq 32 \mathrm{Mpc} / h$, is the same as that for the corresponding fixed mean free path model with $\lambda_{\mathrm{abs}}=32 \mathrm{Mpc} / h, \lambda_{0}=170 \mathrm{Mpc}$.

Fig. 4 shows the ionization field in a $5-\mathrm{Mpc} / h$ slice at $x=0.75$ for $M_{\min }=10^{8} M_{\odot}$, with $\lambda_{\mathrm{abs}}=256 \mathrm{Mpc} / h$ (top) and $\lambda_{\mathrm{abs}}=8 \mathrm{Mpc} / h$ (bottom). The two cases are quite different, with many more small neutral patches for $\lambda_{\mathrm{abs}}=8 \mathrm{Mpc} / h$ relative to $\lambda_{\mathrm{abs}}=256 \mathrm{Mpc} / h$. Also shown in Fig. 4 is the reioniztion redshift in a $0.5-\mathrm{Mpc} / h$ slice. Lower values of $\lambda_{\text {abs }}$ result in a more extended reionization overlap period - i.e. the maxima in reionization redshift are higher, and the minima are lower.

\section{DISCUSSION}

We have carried out large-scale simulations of reionization in a $2 \mathrm{Gpc} / h$ volume, including a finite mean free path to absorption systems. Absorption systems have a significant effect on the characteristic scales at the end of reionization. For the Thomson scattering optical depth reported by WMAP, $\tau_{\text {es }} \simeq 0.09$, we find that the characteristic bubble size when the universe is 90 per cent ionized is quite sensitive to $\lambda_{\mathrm{abs}}$, with $\lambda_{0} \sim 440 \mathrm{Mpc}$ for $\lambda_{\mathrm{abs}}=256 \mathrm{Mpc} / h$ and $M_{\min }=10^{9} M_{\odot}$, while $\lambda_{0} \sim 94 \mathrm{Mpc}$ for $\lambda_{\mathrm{abs}}=8 \mathrm{Mpc} / h$ and $M_{\min }=10^{8} M_{\odot}$.

Calculations using a sharp $k$-space filter lead to a more modest extension of the percolation phase (see Table 1) compared to the reionization history obtained by the solution of equation (1), on which the reionization histories shown in Figure 1 are based. For $\tau_{e s} \simeq 0.09$, the difference in the redshift when the universe is 90 per cent ionized between the $\lambda_{\mathrm{abs}}=$ $8 \mathrm{Mpc} / h$ and $\lambda_{\mathrm{abs}}=256 \mathrm{Mpc} / h$ cases is only $\Delta z_{0.9} \sim 0.3$, while the corresponding case using a sharp real space filter and equation (1) is $\Delta z_{0.9} \sim 0.7$. The delay in the overlap time in both cases is $\Delta z_{\mathrm{ov}} \sim 1.5$.

Using either sharp $k$-space smoothing or equation (1) to model the effect of absorption systems on the reionization history has drawbacks. While equation (1) conserves photons in the long mean free path limit, a mean flux and opacity are assumed, and further work will be necessary to validate and/or improve upon the approach layed out in section 2.1. Using a sharp $k$-space filter results in a reionization history which also conserves photons in the long mean free path limit $\left(k_{F} \longrightarrow 0\right)$, but at the expense of using an oscillatory filter which can "leak" photons from high density regions into lower density ones, and for which there is no unique choice for the relation between $\lambda_{\text {abs }}$ and $k_{F}$. While our results for the timing and photon consumption of the end of reionizaiton are obtained from equations (1) and (9), it is important to note that more work will be necessary to test their accuracy. However, results for the morphology of reionization (Figures 1 and 4) do not depend on the model for the global history, and are therefore more robust.

Our results are consistent with those of Furlanetto \& Oh (2005), in which consumption of ionizing photons in dense systems extends the end of reionization considerably. Choudhury et al. (2009) used the semi-numerical approach in a vol- ume $100 \mathrm{Mpc} / h$ across, and found a similar trend with decreasing $\lambda_{\mathrm{abs}}$, indicating a transition to an "outside-in" morphology near the end of reionization. We find that neutral patches may also remain in voids, where formation of ionizing sources is delayed and radiation from the nearest sources is shielded. Previous studies that modeled the physical origin of absorption systems as minihalos (e.g., Ciardi et al. 2006; McQuinn et al. 2007) also found similar effects to those we find here, although these studies were more focused on the intermediate stages of reionization and on smaller simulated volumes, where the photon mean free path was not as small relative to the bubble sizes as we find in our low mean free path cases in the final, percolation phase.

As mentioned in the introduction, our approach is complementary to that taken by Crociani et al. (2011), in which the semi-numerical approach was used to determine the distribution of absorbers during reionization. They found that their spatial distribution is quite inhomogenous, owing both to intrinsic density fluctuations in the IGM, as well as fluctuations in the ionizing radition field - regions far from sources have a relatively low flux, resulting in a higher abundance of absorbers. This affect was also pointed out by McQuinn et al. (2011), who used high-resolution hydrodynamic simulations post-processed with radiative transfer to determine the mean free path as a function of flux, finding a strongly nonlinear dependence of the mean free path on the emmissivity of ionizing radiation.

These effects imply an important improvement to our model is not only a time-varying mean free path, but also a spatially varying one. To accomplish this, more work will need to be done on the "sub-grid" physics during reionization, using the results of high-resolution cosmological simulations with radiative transfer of a background radiation field, coupled to the hydrodynamics of the gas. However, given that the final patches to be ionized are those most distant from the most luminous sources, the delay in the very end of reionization that we find here is likely to persist when the inhomogeneity of the IGM opacity due to absorption systems is properly taken into account.

The lingering neutral clouds we find would further complicate interpretation of quasar absorption spectra at $z \sim 6$ (e.g., Bolton \& Haehnelt 2007a; Lidz et al. 2007; Alvarez \& Abel 2007; Wyithe et al. 2008; Furlanetto \& Mesinger 2009). As discussed by Mesinger (2009), gaps in quasar spectra can come from either these neutral clouds or mostly ionized but optically-thick absorption systems. The line-of-sight abundance of these neutral clouds at fixed ionized fraction increases with decreasing absorption system mean free path.

As shown by Weinmann et al. (2007) and Alvarez et al. (2009), the Local Group may have been reionized by external sources, i.e. the progenitors of the Virgo Cluster at a distance of $\sim 20 \mathrm{Mpc}$. If $\lambda_{\mathrm{abs}}<20 \mathrm{Mpc}$, however, local reionization would have been delayed until Local Group prongenitors had formed in sufficient number (e.g., Muñoz et al. 2009). Since the satellite population is sensitive to the timing of reionization (Busha et al. 2010), lower values of $\lambda_{\text {abs }}$ could imply a higher local satellite abundance.

Finally, our results have important implications for the interpretation of temperature fluctuations in the cosmic microwave background at multipoles $l \sim 3000$ (Das et al. 2011; Reichardt et al. 2011), through the kinetic SunyaevZel'dovich (kSZ) effect (e.g., McQuinn et al. 2005; Iliev et al. 2007). In particular, Mesinger et al. (2011b) used nearly the same three parameters and approach as we used here for their 

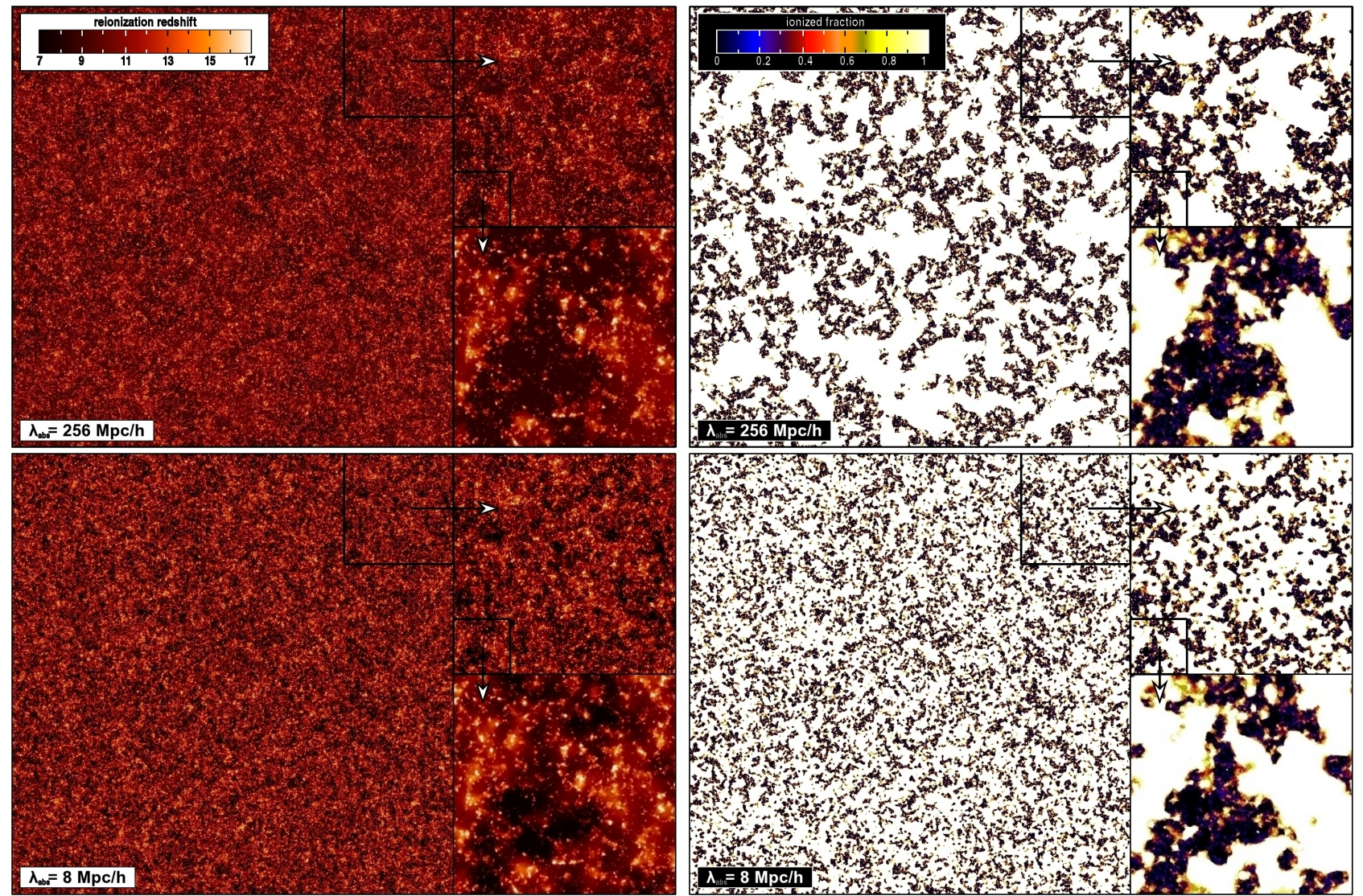

FIG. 4.- left: Reionization redshift in a $0.5 \mathrm{Mpc} / h$-thick slice for the $\lambda_{\mathrm{abs}}=256 \mathrm{Mpc} / h$ simulation (top) and the $\lambda_{\mathrm{abs}}=8 \mathrm{Mpc} / h$ simulation (bottom). Both cases were for $\tau_{\mathrm{es}}=0.09$ and $M_{\min }=10^{8} M_{\odot}$. Clockwise beginning from top-left, regions shown are $2 \mathrm{Gpc} / h, 500 \mathrm{Mpc} / h$, and $125 \mathrm{Mpc} / h$ across. The radii of the circles correspond to $\lambda_{\text {abs. }}$. right: Same as left panels but for the projected ionized fraction in a $5 \mathrm{Mpc} / h$-thick slice at a time when the mean volume-weighted ionized fraction is 0.75 . Note that the ionization field contains considerably more small scale structure in the $\lambda_{\text {abs }}=8 \mathrm{Mpc} / h$ case.

parameter study of the dependence of the kSZ signal on the reionization scenario. Reducing the mean free path shifts power to higher multipoles because of the accompanying decrease in the bubble size that we find. Although the kSZ effect is only just beginning to constrain the duration of reionization (Zahn et al. 2011b), more detailed future observations and analysis will begin to provide constraints on the patchiness in addition, and understanding the effect of absorption systems on reionization will be crucial.

We thank M. Haehnelt, M. McQuinn, A. Mesinger,
R. Thomas, G. Vasil for useful discussions, and J. Chluba for providing the diffusion solver from CosmoRec. The simulations in this paper were performed on the GPC supercomputer at the SciNet HPC Consortium. SciNet is funded by: the Canada Foundation for Innovation under the auspices of Compute Canada; the Government of Ontario; Ontario Research Fund - Research Excellence; and the University of Toronto. This work was partially supported by NASA ATFP grant NNX08AH26G, NSF AST-0807312, and NSF AST0808398 .

\section{REFERENCES}

Abel, T. \& Mo, H. J., 1998, ApJ494, 151

Abel, T., Norman, M. L., \& Madau, P. 1999, ApJ, 523, 66

Abel, T. \& Wandelt, B. D. 2002, MNRAS, 330, L53

Alvarez, M. A. \& Abel, T. 2007, MNRAS, 380, L30

Alvarez, M. A., Busha, M., Abel, T., \& Wechsler, R. H. 2009, ApJ, 703, L167

Bolton, J. S. \& Haehnelt, M. G. 2007b, MNRAS, 382, 325

-. 2007a, MNRAS, 374, 493

Bond, J. R., Cole, S., Efstathiou, G., Kaiser, N. 1991, ApJ, 379, 440

Busha, M. T., Alvarez, M. A., Wechsler, R. H., Abel, T., \& Strigari, L. E. 2010, ApJ, 710, 408

Choudhury, T. R., Haehnelt, M. G., \& Regan, J. 2009, MNRAS, 394, 960

Ciardi, B., Scannapieco, E., Stoehr, F., Ferrara, A., Iliev, I. T., \& Shapiro, P. R. 2006, MNRAS, 366, 689

Crociani, D., Mesinger, A., Moscardini, L., Furlanetto, S., 2011, MNRAS, 411,289

Das, S., et al., 2011, ApJ, 729, 62
Dijkstra, M., Haiman, Z., Rees, M. J., Weinberg, D. H., 2004, ApJ, 601, 666 Friedrich, M. M., Mellema, G., Alvarez, M. A., Shapiro, P. R., Iliev, I. T., 2011, MNRAS, 413, 1353

Furlanetto, S. R. \& Mesinger, A. 2009, MNRAS, 394, 1667

Furlanetto, S. R. \& Oh, S. P. 2005, MNRAS, 363, 1031

Furlanetto, S. R., Oh, S. P., \& Briggs, F. H. 2006, Phys. Rep., 433, 181 Furlanetto, S. R., Zaldarriaga, M., \& Hernquist, L. 2004, ApJ, 613, 1

Gnedin, N. Y. 2000, ApJ, 542, 535

Gnedin, N. Y. \& Abel, T. 2001, New Astronomy, 6, 437

Gnedin, N. Y. \& Fan, X. 2006, ApJ, 648, 1

Haiman, Z., Abel, T., \& Madau, P. 2001, ApJ, 551, 599

Iliev I. T., Pen U.-L., Bond J. R., Mellema G., Shapiro P. R., 2007, ApJ, 660, 933

Iliev, I. T., Mellema, G., Pen, U.-L., Merz, H., Shapiro, P. R., \& Alvarez, M. A. 2006, MNRAS, 369, 1625

Komatsu, E. et al. 2011, ApJS, 192, 18 
Lidz, A., McQuinn, M., Zaldarriaga, M., Hernquist, L., \& Dutta, S. 2007, ApJ, 670, 39

McQuinn M., Furlanetto S. R., Hernquist L., Zahn O., Zaldarriaga M., 2005, ApJ, 630, 643

McQuinn, M., Lidz, A., Zahn, O., Dutta, S., Hernquist, L., \& Zaldarriaga, M. 2007, MNRAS, 377, 1043

McQuinn, M., Oh, S. P., Faucher-Giguere, C., 2011, ApJ, 743, 82

Mesinger, A. 2009, MNRAS, submitted, arXiv:0910.4161

Mesinger, A. \& Furlanetto, S. 2007, ApJ, 669, 663

Mesinger, A., Furlanetto, S., \& Cen, R. 2011, MNRAS, 411, 955

Mesinger, A., McQuinn, M., Spergel, D. N., 2011, MNRAS, submitted, arXiv: 1112.1820

Miralda-Escudé, J., Haehnelt, M., Rees, M. J. 2000, ApJ, 530, 1

Mo, H. \& Miralda-Escudé, J., 1996, ApJ, 469, 589

Muñoz, J. A., Madau, P., Loeb, A., \& Diemand, J. 2009, MNRAS, 400, 1593

Pawlik, A. H., Schaye, J., van Scherpenzeel, E., 2009, MNRAS, 394, 1812

Prochaska, J. X., O’Meara, J. M. \& Worseck, G. 2010, ApJ, 718, 392

Prochaska, J. X., O'Meara, J. M., \& Worseck, G. 2009, ApJ, 705, 113

Raicevic, M. \& Theuns, T., 2011, MNRAS, 412, 16

Reichardt, C. L., et al., 2011, ApJ, submitted, arXiv:1111.0932

Shapiro, P. R., Iliev, I. T., \& Raga, A. C. 2004, MNRAS, 348, 753
Santos, M. G., Ferramacho, L., Silva, M. B., Amblard, A., Cooray, A., 2010, MNRAS, 406, 2421

Shin, M., Trac, H., Cen, R., 2008, ApJ, 681, 756

Songaila, A. \& Cowie, L. L. 2010, ApJ, 721, 1448

Sokasian, A., Abel, T., \& Hernquist, L. E. 2001, New Astronomy, 6, 359

Storrie-Lombardi, L. J., McMahon, R. G., Irwin, M. J., \& Hazard, C. 1994, ApJ, 427, L13

Thomas, R. M., et al., 2009, MNRAS, 393, 32

Trac, H. \& Cen, R. 2007, ApJ, 671, 1

Tytler, D., 1982, Nature, 298, 427

Weinmann, S. M., Macciò, A. V., Iliev, I. T., Mellema, G., \& Moore, B. 2007, MNRAS, 381, 367

Weymann, R. J., Carswell, R. F., Smith, M. G., 1981, ARA\&A, 19, 41

Wyithe, J. S. B., Bolton, J. S., \& Haehnelt, M. G. 2008, MNRAS, 383, 691

Zahn, O., Lidz, A., McQuinn, M., Dutta, S., Hernquist, L., Zaldarriaga, M., \& Furlanetto, S. R. 2007, ApJ, 654, 12

Zahn, O., Mesinger, A., McQuinn, M., Trac, H., Cen, R., \& Hernquist, L. E., 2011a, MNRAS, 414, 727

Zahn, O., et al., 2011b, ApJ, submitted, arXiv:1111.6386 